\title{
Periprosthetic Femoral Fractures in Total Knee Arthroplasty
}

\author{
Vladan Stevanović, Zoran Vukašinović, \\ Zoran Baščarević, Branislav Starčević, \\ Dragana Matanović and Duško Spasovski \\ Additional information is available at the end of the chapter \\ http://dx.doi.org/10.5772/55226
}

\section{Introduction}

Total joint arthroplasty has greatly improved the treatment of knee arthrosis, but still is not without complications. Supracondylar fractures above total knee replacements are an uncommon complication (incidence $0,3 \%$ to $2.5 \%$ ), occuring more frequently in patients older than 60 years with osteoporotic bone. The rate of these fractures is expected to increase in the future because of the growing number of total knee replacements and greater level of acitivity among elderly patients. The timing of such fractures has been reported to range from early in the postoperative period to more than a decade after surgery, with a mean of 2 to 4 years. During the past two decades authors were not agreed in the definition of periprosthetic supracondylar region: the lower 3 inches $(7 \mathrm{~cm})$ of the femur [1]; $9 \mathrm{~cm}$ proximal to the knee joint line [2]; all fractures within $15 \mathrm{~cm}$ proximal to the knee joint line [3]. Generally, based on the older literature, supracondylar periprosthetic fractures were those within $15 \mathrm{~cm}$ of the joint line, or in the case of stemmed component, within $5 \mathrm{~cm}$ of the proximal end of the implant. Nevertheless, the most important is understanding that these fractures occur in regions of stress concentration adjacent to a prosthetic component, and that the presence of the prosthesis has a significant effect on fracture treatment. So, we suggest that fractures above total knee replacement should be considered supracondylar fractures if they extend within $7 \mathrm{~cm}$ of the prosthetic joint line or if they are within $2 \mathrm{~cm}$ of the femoral prosthetic flange.

The most commonly suggested predisposing factors for a periprosthetic femoral fracture after total knee arthroplasty are osteopenia, revision arthroplasty, rheumatoid arthritis, use of steroids, existing neurological disorders, misalignment of the components, and notching of 
the anterior femoral cortex. Different factors were found in the pathogenesis of the fracture: stress-shielding from the anterior flange of the femoral component, inadequate osseous remodeling due to postoperative hypovascularity, relative difference in elastic modulus between the implant-covered distal part of the femur and femoral cortex, endosteal ischemia from metal or bone cement, and osteolysis of the distal part of the femur secondary to polyethylene wear debris. The majority of these fractures results from a combination of axial and torsion loads. Most of them occur following minimal falls, while the rest of them are secondary to motor-vehicle accidents, seizers or closed manipulation of a stiff knee after total knee arthroplasty

\section{Prevalence and pathogenesis}

The prevalence of supracondylar femoral fracture in patients with total knee replacement ranges from 0.3 to $4.2 \%$. Most of the patients who sustain fractures about a total knee arthroplasty are women, usually in their seventh decade of life. As with other supracondylar fractures in the elderly, periprosthetic fractures usually occurs after low energy trauma. Osteoporosis is often present as well, due to a number of factors including stress shielding because of a rigid implant, pharmacologic causes, hormonal influences and senility. An association with rheumatoid arthritis, especially when the patient is receiving oral corticosteroid treatment, has been noted. Neurologic disorders have also been involved in the occurrence of these fractures, due to either medication induced osteoporosis or gait disturbance. In addition, revision arthroplasty has been associated with an increased incidence of periprosthetic fractures, more commonly when constrained implants are used, as they transfer applied torque more directly to bone that is potentially already deficient. Notching of the anterior femoral cortex during total knee arthroplasty has been indicated as one factor contributing to these periprosthetic femoral fractures. The prevalence of inadvertent cortical notching of the femur during total knee arthroplasty has been reported to be as high as $27 \%$ and there are several studies performed to quantify the reduction in bending and torsion strength resulting from femoral notching in attempt to provide the clinician with useful information related to the postoperative management [5,6]. Clearly, notching of the anterior femoral cortex is neither the only risk factor nor the principal risk factor for supracondylar femoral fracture after knee replacement. Of a total of 6470 total knee arthroplasties included in reports on this subject, only seventeen $(0.26 \%$ ) were complicated by a supracondylar femoral fracture associated with anterior notching compared with nearly three times as many fractures that occurred in the absence of notching [5]; biomechanical effects of femoral notching following total knee arthroplasty showed mean decrease in bending strength of $18 \%$ (8-31\%) and mean reduction in torsion strength of $39.2 \%(19-73 \%)$ in cadaveric specimens [6]. Based on Wolff's law, distal part of the femur would strengthen after the operation as result of remodeling, thus reduction in femoral bone strength should primarily be expected in the immediate postoperative period. Therefore a clear recommendation should be given to the patients who sustain inadvertent notching that they should have additional protection in the early postoperative period, and to consider the use a femoral component with stem as a means to bypass the stress riser of the 
anterior cortical notch. Most important, authors believe that an anterior cortical notch should be considered as a contraindication for manipulation of the knee prosthesis in the early postoperative period $[7,8]$.

Anterior defects may be present without notching, such as in cases of cystic lesions of degenerative or rheumatoid origin near the proximal aspect of the anterior femoral flange. Adequate remodeling may not be possible after those cysts are filled with cement at the time of arthroplasty. These defects remain as permanent stress risers, which may predispose to fracture. Large anterior effects might be better managed during primary knee arthroplasty with bone grafting and protection of the distal femur with an intramedullary stem [9].

Another recently recognized factor leading to late supracondylar femoral fracture is the presence of a massive debris-related osteolytic defect in the distal femur; such defects have been reported in association with asymptomatic well-fixed cementless femoral component. Ankylosis of a total knee arthroplasty may also predispose a fracture by producing increased stress in the distal femoral metaphysis $[10,11]$.

\section{Risk factors / etiology}

Literature data show that patients with osteopenia are at greater risk to acquire supracondylar femoral fracture after total knee arthroplasty, followed by rheumatoid arthritis, corticosteroid treatment, female gender and older age $[12,13,14]$. Additional risk factors are: neurological disorders, a revision total knee replacement (TKR) and rotationally constrained implants that create increased torsion load transfer to bone [15] (Table1).

\footnotetext{
Osteopenia

Rheumatoid arthritis

Steroid use

Neurologic disorders

Revision TKR

Female gender

Seventh decade of life

Distal femoral osteolysis

Anterior femoral notching +/-
}

Table 1. Risk factors for supracondylar femoral fractures, in decreasing order

Clinical and biomechanical data on anterior notching of the distal femoral cortex confirm the increase of fracture risk, and theoretical mathematical analysis calculated that a three-millimeter notch results in a $30 \%$ reduction in torsion bone strength [9]. On the other hand, a series of 670 total knee prosthesis with $20 \%$ femurs with anterior notching of at $3 \mathrm{~mm}$ at least, and found only two supracondylar fractures [11]. Different fracture patterns are associated with notched and no notched femurs: notched femurstend tohaveshortobliquefracturesoriginating from thenotched cortex, whereas no notched femurs tend to have diaphyseal fractures. 
Furthermore, there is a general feeling that the most significant risk factor causing supracondylar fracture is the increase in activity that elderly patients achieve after knee replacement, exposing them to a greater risk of slipping and falling.

\section{Diagnostic algorithm}

Patients with this type of injury usually provide a history of minor trauma, such as fall during ambulation. They usually present with pain and inability to bear weight. Since these are typically low energy injuries, major tissue swelling is uncommon. Unless marked displacement is present, deformity may not be apparent on examination.

A thorough evaluation includes careful physical examination, a review of the patient's medical history and adequate radiographic studies. The injured limb should be assessed for soft tissue integrity and neurovascular status. The location of previous skin incisions must also be noted.

A complete radiographic examination of a fracture about a total knee arthroplasty includes standard anteroposterior and lateral radiographs as well as long leg views of the involved limb; oblique images and tomography are also often useful (Table 2). The diagnostic evaluation must include a direct lateral view of the distal femur in order to guide subsequent treatment: the direct lateral view facilitates assessment of fracture displacement, while also revealing the bone available for fixation devices, the location of femoral lugs of posterior cruciate retaining components and the proximal extent of the central femoral recess in cases with posterior stabilized components. Radiographically, nondisplaced or minimally displaced fractures may be obscured by the femoral flange; it is important to identify nondisplaced fractures since displacement may occur later.

Fracture displacement and comminution

Axial limb alignment

Quality of bone stock

Location of the fracture relative to the prosthesis

Stability of the prosthesis

Table 2. Characteristics of radiography assessment

Review of prefracture radiographs can provide important data regarding baseline limb alignment, implant fixation and the presence of regions of osteolysis or polyethylene wear. The type and technical specifications of the implant and templates in place will influence the selection of fixation device if open reduction is necessary [16, 17].

The first step is to establish whether the implant is loose; if so even if the fracture is well aligned and heals, treatment that does not include revision will lead to poor result. Prefracture misalignment, osteolysis and polyethylene wear are important factors in the decision making process. 
The second step in the treatment is to identify fracture displacement and to decide whether reductionisneeded.Anyalterationinlimbaxisresulting fromfracturecanresultinaltered loading of the prosthesis, which may in turn lead to enhanced wear and/or accelerated implantloosening. The third step is to determine the appropriate treatment for displaced fracture (Figure 1).

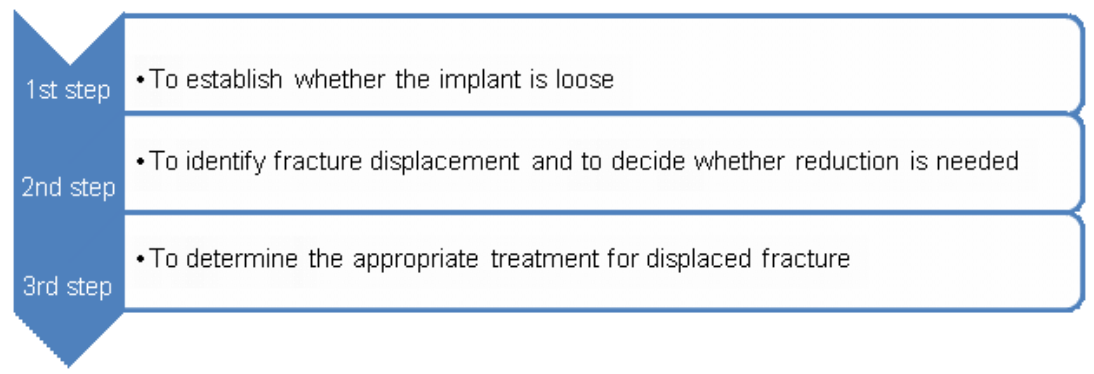

Figure 1. Diagnostic algorithm for periprosthetic supracondylar femoral fracture above total knee arthroplasty

\section{Classification}

Numerous systems of classification of supracondylar femoral fractures after total knee arthroplasty have been developed. Most of the classifications were based on supracondylar fractures without knee arthroplasty (Neer et al, DiGioia and Rubash, Chen et al.) (Table 3).

\begin{tabular}{|c|c|c|}
\hline \multirow[t]{5}{*}{ Neer et al. } & Type I & $\begin{array}{r}\text { Undisplaced }\left(<5 \mathrm{~mm} \text { displacement or }<5^{0}\right. \\
\text { angulation })^{*}\end{array}$ \\
\hline & Type II & Displaced $>1 \mathrm{~cm}$ \\
\hline & Type Ila & With lateral femoral shaft displacement \\
\hline & Type IIb & With medial femoral shaft displacement \\
\hline & Type III & Displaced and comminuted \\
\hline \multirow[t]{3}{*}{ DiGioia and Rubash } & Group I & Extraarticular, undisplaced* \\
\hline & Group II & Extraarticular, displaced* \\
\hline & Group III & $\begin{array}{r}\text { Severely displaced (loss of cortical contact) or } \\
\text { angulated }\end{array}$ \\
\hline \multirow[t]{2}{*}{ Chen et al } & Type I & Nondisplaced(Neer I) \\
\hline & Type II & Displaced or comminuted (Neer I or II) \\
\hline
\end{tabular}

Table 3. Classification of supracondylar femoral fractures above total knee arthroplasty reprinted from Su ET, De Wal H, Di Cesare P. Periprosthetic Femoral Fractures Above Total Knee Replacements J Am Acad Orthop Surg, 2004; $12: 12$ - 20. - with permission (personal communication)

For identifying fracture displacement and deciding whether reduction is needed Rorabeck et al. [18] created classification that takes into account both the status of the prosthesis (intact or failing) and the displacement of the fracture: 
Type I: fracture is undisplaced and the prosthesis is intact; Type II: fracture is displaced and the prosthesis is intact; Type III: fracture is displaced or undisplaced and the prosthesis is loose or failing

Summarizing above mentioned classifications, we strongly support suggested and explained in article by Su et al.[4] which is transcripted (Figure 2)
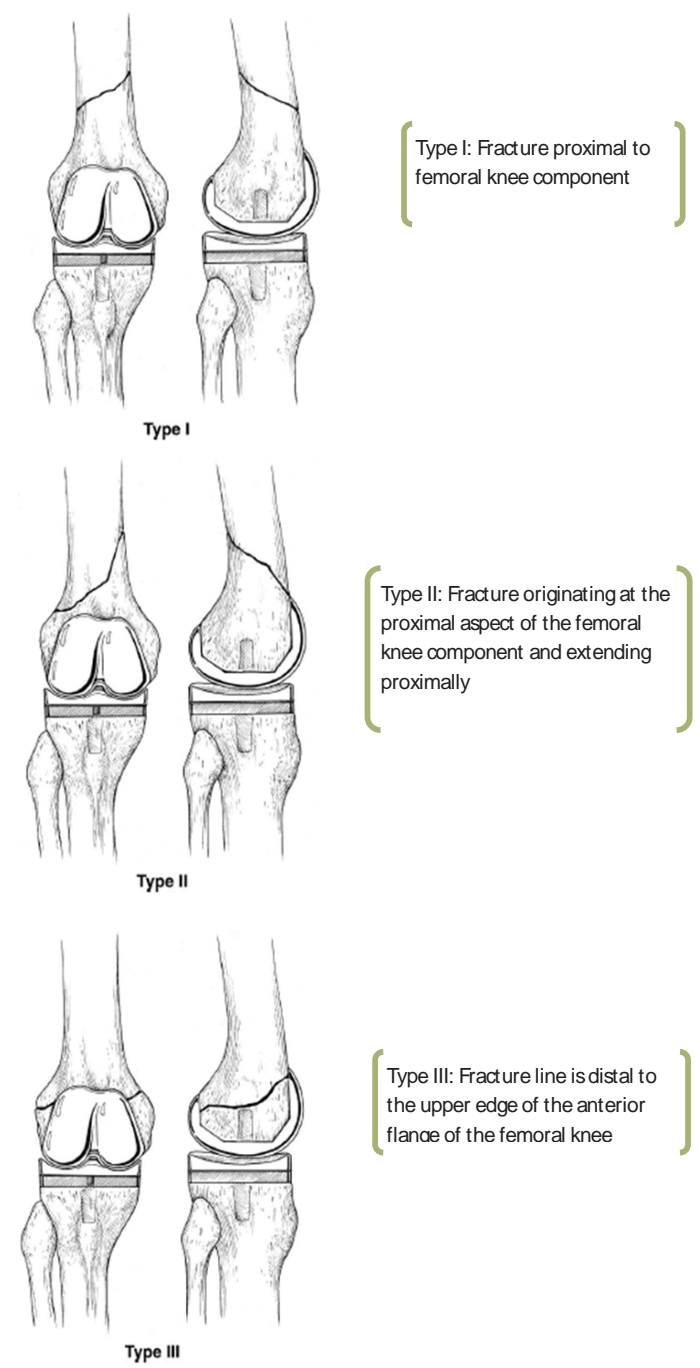

Figure 2. Reprinted from Su ET, De Wal H, Di Cesare P. Periprosthetic Femoral Fractures Above Total Knee Replacements J Am Acad Orthop Surg, 2004; 12:12 - 20. - with permission (personal communication) 


\section{Treatment options}

The treatment of supracondylar fractures of the femur following total knee replacement has been a challenge for the orthopaedic surgeon, regardless the fracture and type of fixation [19, 20, 21, 22]. The major goal of treatment should be the restoration of the prefracture functional status of the patient which is characterized by: fracture union, preservation of prosthetic components without loosening, infection and other complications, maintenance of appropriate prosthetic alignment, restoration of joint range of motion. The need to meet all of these objectives makes these fractures difficult to treat: if even single goal is not achieved, the results of treatment will be suboptimal and may lead to failure of the prosthesis. There are two main treatment options: closed (without implant revision) or open (with implant revision), each with various modalities (Tables 4,5) [23, 24, 25, 26].

Skeletal traction

Application of a cast

Pins and plaster

Cast bracing

Table 4. Options for closed treatment of periprosthetic supracondylar femoral fractures

Use of condylar plate

Intramedullary fixation (flexible or rigid, interlocking)

Revision total knee arthroplasty

External fixation

Cerclage wiring with strut allograft fixation

Arthrodesis

Table 5. Options for open treatment of periprosthetic supracondylar femoral fractures

While fracture configuration influences the choice of open versus closed treatment method, fracture displacement, the degree of osteopenia, and the type and technical specifications of the prosthetic components are most valuable determinants of the operative fracture management. Treatment results are closely associated with postfracture alignment and stability [27]. Fracture displacement, intercondylar extension and comminution are negative prognostic factors. High malunion rates are common in association with varus, flexion and internal rotation deformities typically seen as a result of forces exerted by the adductor and gastrocnemius muscle group. Varus femoral malunion is associated with a risk of premature failure of the total knee arthroplasty. The choice of operative treatment method should be based on the patient's health, fracture configuration and displacement, presence of comminution, severity of osteopenia and status of the prosthetic components (loose, unstable or malaligned)[28, 29]. 


\section{Nonoperative treatment}

The advantages of nonoperative treatment are: noninvasiveness and negligible infection rate. Since fracture union is likely in nondisplaced fractures, nonoperative treatment is uniformly recommended as the initial management in these cases. Disadvantages include: a relatively high malunion rate and functional loss, particularly in patients with displaced fracture through osteopenic bone in whom maintance of reduction is difficult. Nonoperative treatment is best reserved for nondisplaced fractures that do not demonstrate intercondylar extension. Nonsurgical management does eliminate surgical risks such as bleeding, infection, loss of fixation and anesthetic complications. On the other hands, prolonged recumbency in elderly patients carries the significant risk of decubitus ulcers, pneumonia, pulmonary embolia, deep venous thrombosis and diffuse muscle atrophy [30].

\section{Operative treatment}

Management of periprosthetic fractures of the femur above total knee arthroplasty depends on displacement at the fracture site, bone quality, size of distal fragment and condition of implants (Table 6) [31, 32, 33, 34].

\begin{tabular}{lll}
\hline Fracture type & Description of fracture & Treatment recommendation \\
\hline I & Undisplaced fracture and well fixed prosthesis & Bracing, nonweightbearing \\
\hline II & Displaced fracture and well fixed prosthesis & Internal fixation using conventional \\
& plate, intramedullary nail or locking \\
& Poor quality bone with osteopenia and & plate \\
& comminution & Intramedullary nail or locking plate \\
\cline { 2 - 3 } & Decent size distal fragment & Locking plate or buttress plate with \\
& Extremely distal fracture & strut allograft \\
\hline III & Displaced fracture, loose prosthesis & Revision knee arthroplasty using a \\
& No metahyseal bone loss & long stemmed femoral implant \\
\hline & Metaphyseal bone loss or nonunion following & Structural allograft prosthesis \\
& previous surgery & composite or distal femoral \\
& & replacement prosthesis \\
\hline
\end{tabular}

Table 6. Operative guidelines for the treatment of periprosthetic supracondylar fractures above total knee arthroplasty

Open reduction and fixation with a condylar plate provides the potential advantages of anatomical reconstruction, rigid fixation and an early range of motion exercise. Maintenance of reduction can be a problem, particularly when a patient has a comminuted fracture through osteopenic bone, and malunion is commonly observed. Use of condylar plate is best reserved for less comminuted, displaced fractures with satisfactory bone stock. Using the buttress condylar plate 
include the ability to place the multiple screws distally in many directions and excellent visualization of thefracture to obtain an anatomicreduction. Disadvantagesincludeextensivesoft tissue stripping and less rigid fixation than with a nail or fixed angle condylar plate.

Use of flexible intramedullary rods is an efficient and less invasive treatment option, although shortening and rotational malunion occasionally occur as a result of the reduced axial and rotational stability. This technique should be considered for mildly displaced fractures patients with unstable general condition. It is minimally invasive procedure with limited morbidity.

New locking plates offer advantages over conventional plates for the treatment of periprosthetic fracture associated with total knee arthroplasty. These devices provide stable fixation in osteopenic bone, they are adaptable to different types of fracture and prosthesis and can be inserted using a minimally invasive approach. These plates are particularly useful in presence of an implant in proximal femur as it allows unicortical screw fixation if there is overlapping the distal part of the proximal implant, thus avoiding a stress riser between the two implants.

Rigidsupracondylarinterlocking rod fixation offers the advantage of being minimally invasive while providing good axial, angular and rotational stability. It can be performed with use of minimal patellar tendon splitting approach with percutaneous placement of interlocking screws in cases with lesser comminution with maintenance of the fracture hematoma and osseous blood supply. Contraindications include loose total knee components, severe comminution, extremely distal fracture and a presence of long total hip intramedullary stem,. This technique has several advantages over traditional open reduction with plate fixation: intramedullary implants are biomechanically superior to subperiostally placed fixation devices, whohave significantly larger bending moments; there is no need for periosteal stripping, which can compromise blood supply to the fracture site and increase the risk of nonunion; plate fixation can be technically demanding and often requires the use of supplemental bone grafting.

Revision total knee arthroplasty provides the advantage of stable fixation with a dyaphisis engaging intramedullary femoral stem, allowing early range of motion and weight bearing. This technique is selected for extremely distal or comminuted fractures when stable fixation is difficult to secure with other methods, or for any fracture associated with loose, unstable, or substantially malaligned total knee components. Revision total knee arthroplasty is frequently required in cases where other methods, nonoperative or operative, have failed.

The most difficult cases involve a loose prosthesis coupled with deficient metaphyseal bone stock, rendering a basic revision procedure impossible. Such cases require excision of distal fracture fragment and replacement with either a distal femoral replacement prosthesis or a structural allograft. These treatment methods may also be required for nonunion resulting from failed osteosynthesis. Distal femoral replacement implants should be considered as a limb salvage option when other surgical options are not feasible. The use of stemmed constrained revision component with structural distal femoral allograft composite has been described as the effective means of providing both implant and fracture stability.

Periprosthetic fractures have a higher rate of nonunion than other supracondylar femoral fractures in the elderly. This has been attributed to alterations in vascularity at the fracture site 
due to primary surgery, the presence of metal implant and intramedullary polymethyl methacrylate (PMMA), or long term oral corticosteroid administration.

The goals of treatment, whether surgical or nonsurgical, are fracture healing, restoration and maintenance of knee range of motion, and pain free ambulation. A good result is a minimum of 90 degrees of knee motion, with femoral shortening less then $2 \mathrm{~cm}$, varus/valgus malalignment less than 5 degrees, and flexion/extension malalignment less than 10 degrees. Fulfillment of these criteria enables satisfactory knee function, which is of paramout importance to the patient.

\section{Complications}

Major early complications include nonunion and malunion, which often lead to prosthetic loosening, pain and revision. The treatment of delayed unions with bone grafting is possible and is advocated if appropriate limb alignment and fracture fixation are maintained. In cases of deformity, early signs of prosthetic failure or inability to secure rigid fixation, revision may be the most appropriate. The most devastating complication of operative care of these fractures is infection. Incidence of periprosthetic fracture following total knee arthroplasty is gradually increasing, and management of these fractures can be challenging with complications that severely influence both the patient and surgeon. Furthermore, treatment complication rate range from 20 to 75 percent according to literature data [35]: in a review of 415 cases, there were reported a nonunion rate of $9 \%$, fixation failure in $4 \%$, an infection rate of $3 \%$ and revision surgery rate of $13 \%$. Following case will demonstrate some of these problems in treating supracondylar periprosthetic femoral fractures.
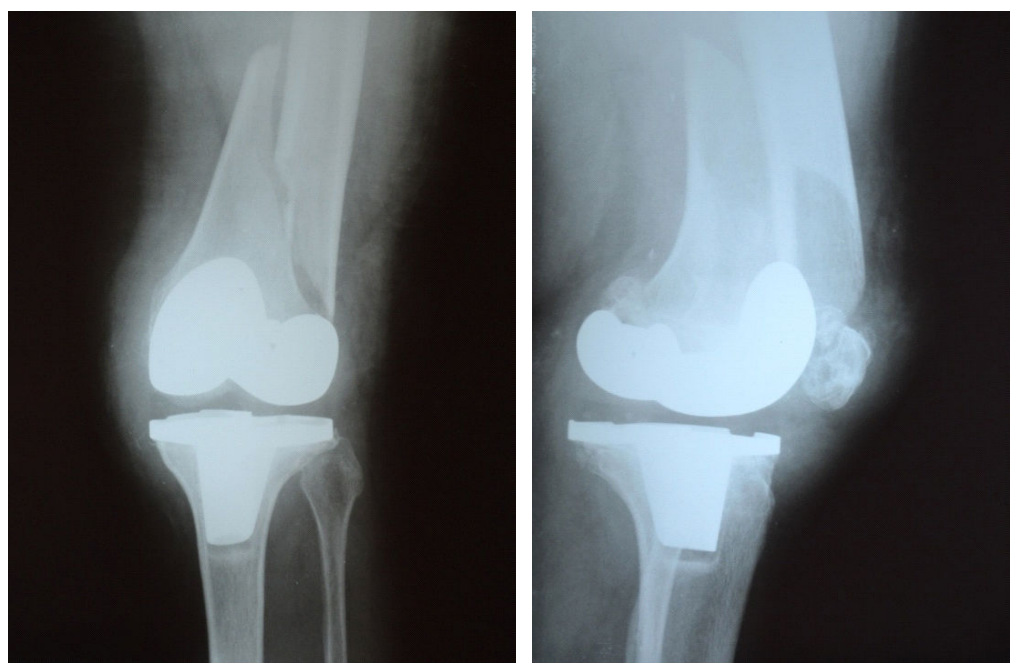

Figure 3. Anteroposterior and lateral view of type II supracondylar femoral fracture 


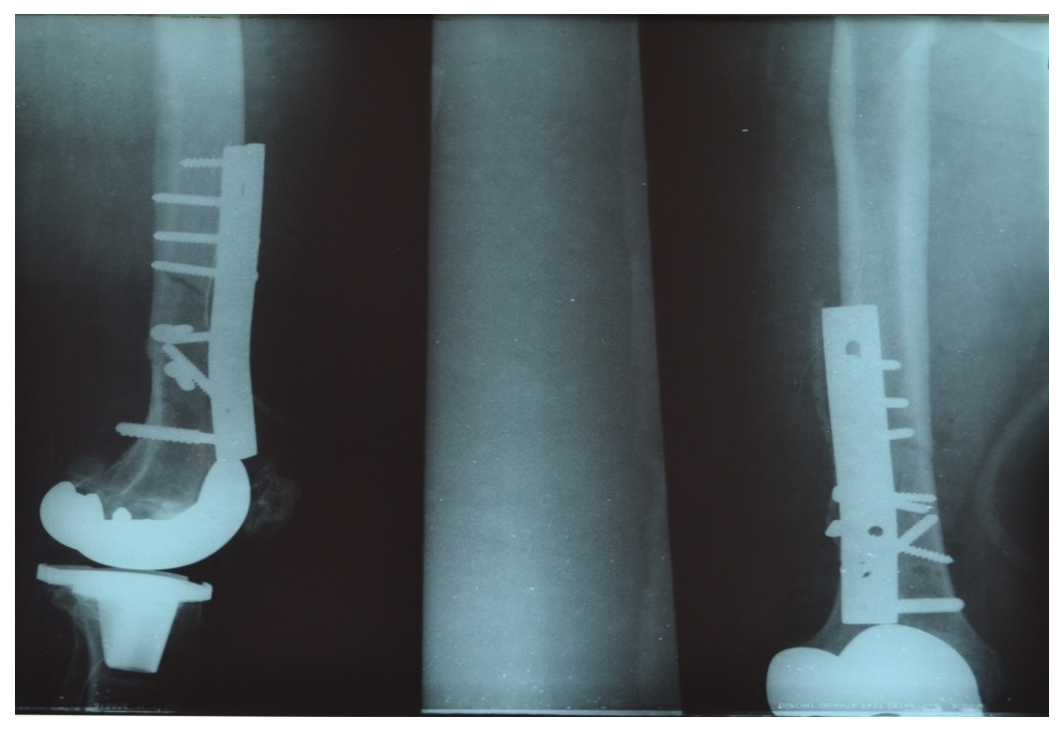

Figure 4. Operative treatment with DCP (anatomic reduction with rigid fixation)

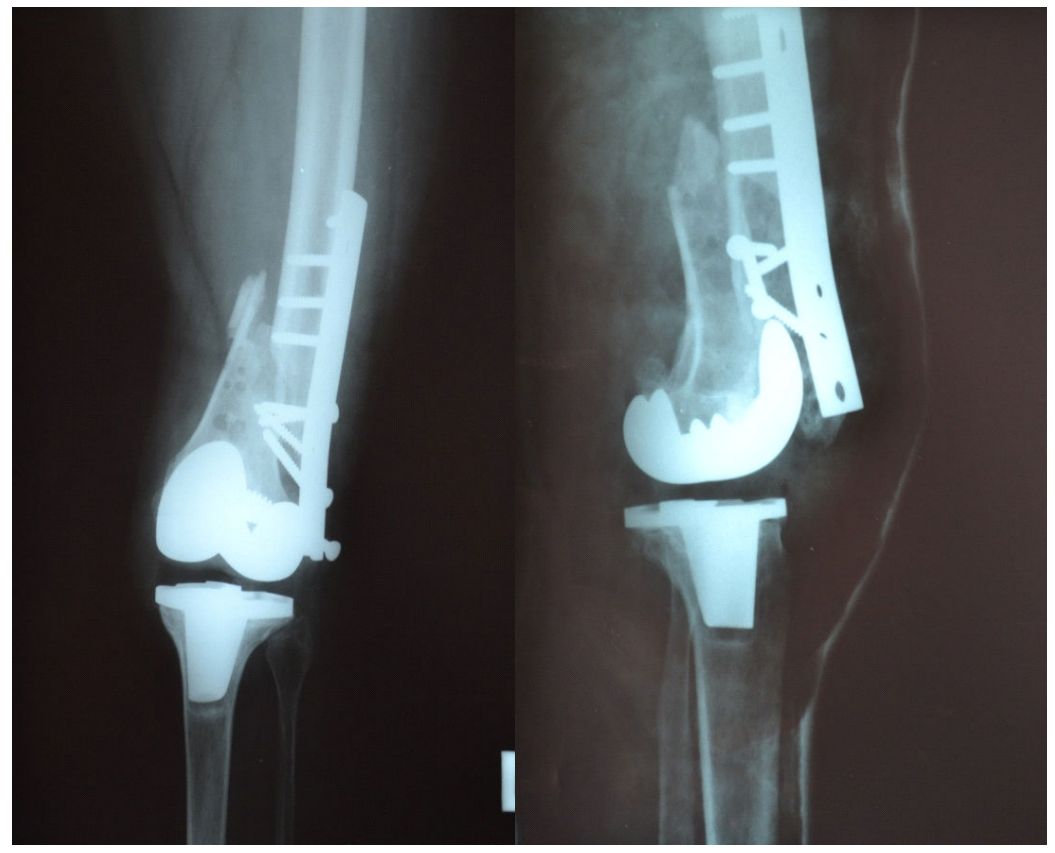

Figure 5. Loss of reduction and fixation two months following surgery 


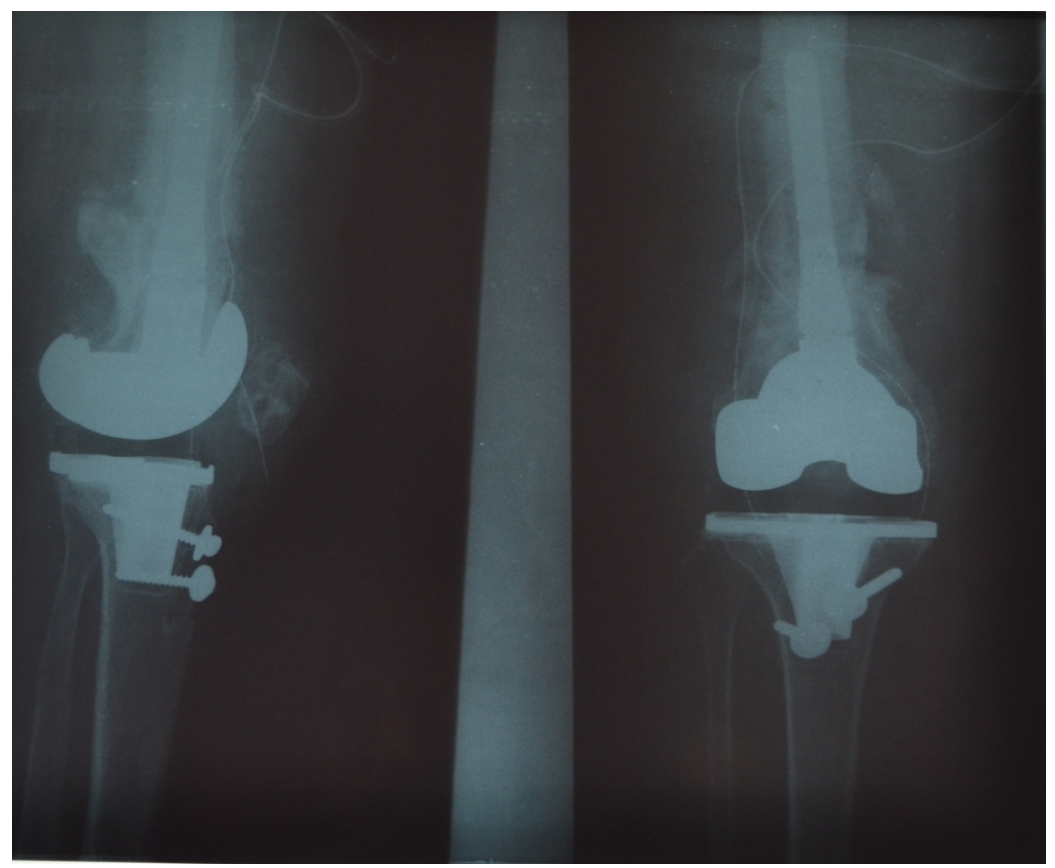

Figure 6. Revision total knee arthroplasty for loose femoral component and fracture treatment

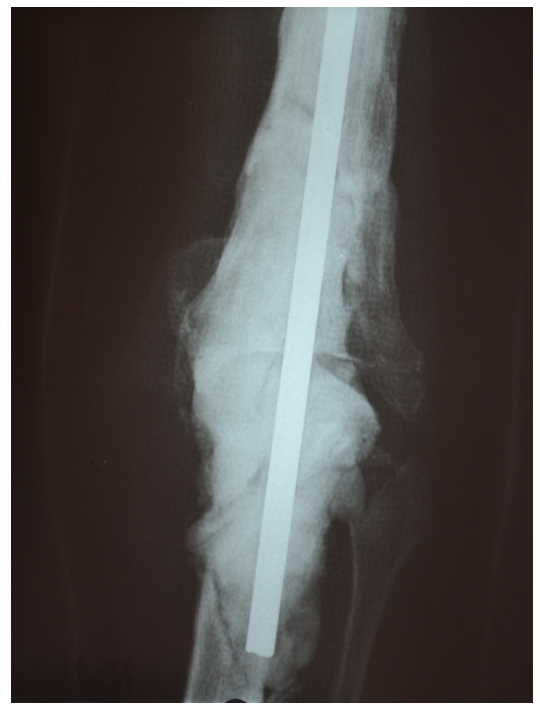

Figure 7. Devastating complication, infection, and limb salvage procedure with antibiotic cement spacer 


\section{Aftertreatment, rehabilitation}

Rehabilitation process is generally guided by the characteristics of the fracture and chosen treatmen methods. As previously said, non-operative treatment is reserved for nondisplaced supracondylar fractures with stable implant and includes longer rehabilitation period to achieve patient's preambulatory status, if it is possible at all. Since surgery and more stable implants including intramedullary nails and angular locking plates allow for faster aftertreatment program, rehabilitation protocol is similar to post fracture treatment in cases without knee arthroplasty. Main goals are fracture healing, implant stability and prefracture functional status.

\section{Prevention}

Since supracondylar femoral fractures above total knee arthroplasty are mostly seen in osteoporotic patients, prevention of osteopenia and osteoporosis including treatment with bisphosphonate and regular exercise will be good for the well-being of the patient and implant. Surgical factors, such as anterior femoral notching, bone cement hypovascularisation and termal necrosis, and uncontrolled soft tissue manipulation should be kept in mind on regular basis in order to minimize surgeon's impact on development of potential complications including supracondylar fracture.

\section{Conclusion}

Periprosthetic femoral fractures above total knee prosthesis are increasing complication with constantly growing incidence since the number of total knee replacements and population agings are convergating factors. Risk factors analysis and prevention should be in surgeon and patient focus on this topic. Treatment options include first step to establish whether the implant is loose and, if so even if the fracture is well aligned and heals, treatment that does not include revision will lead to poor result. Prefracture misalignment, osteolysis and polyethylene wear are important factors in the decision making process. The second step in the treatment is to identify fracture displacement and to decide whether reduction is needed. The most appropriate criteria of acceptable fracture alignment are for supracondylar fractures without knee prosthesis: less than $5 \mathrm{~mm}$ of translation; less than 5 to10 degrees of angulation; less than 10 $\mathrm{mm}$ of shortening and less than 10 degrees of rotational displacement. Any alteration in limb axis resulting from fracture can result in altered loading of the prosthesis, which may in turn lead to enhanced wear and/or accelerated implant loosening. The goals of treatment, whether surgical or nonsurgical, are fracture healing, restoration and maintenance of knee range of motion, and pain free ambulation. A good result is a minimum of 90 degrees of knee motion, with femoral shortening less then $2 \mathrm{~cm}$, varus/valgus malalignment less than 5 degrees, and flexion/extension malalignment less than 10 degrees. Fulfillment of these criteria enables satisfactory knee function, which is of paramout importance to the patient. 


\section{Acknowledgements}

This work is supported by grant number III 41004, Ministry of Education and Science Republic of Serbia.

\section{Author details}

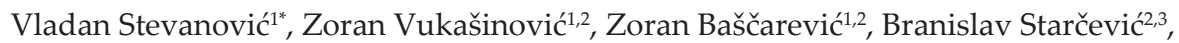
Dragana Matanovićc, ${ }^{2}$ and Duško Spasovski ${ }^{1,2}$

*Address all correspondence to: vladanstevanovi90@gmail.com

1 Institute for Orthopaedic Surgery „Banjica“, Belgrade, Serbia

2 Faculty of Medicine, University of Belgrade, Belgrade, Serbia

3 Clinic for Orthopaedic Surgery and Traumatology, Clinical Center of Serbia, Belgrade, Serbia

4 Clinic for Physical Therapy and Rehabilitation, Clinical Center of Serbia, Belgrade, Serbia

\section{References}

[1] Neer, C. S I. I, Grantham, S. A, \& Shelton, M. L. Supracondylar fracture of the adult femur: A study of one hundred and ten cases. J Bone Joint Surge Am (1967). , 49, 591-613.

[2] Culp, R. W, Schmidt, R. G, Hanks, G, \& Mak, A. Esterhai JL Jr, Heppenstall RB. Supracondylar fracture of the femur following prosthetic knee arthroplasty. Clin Orthop (1987). , 222, 212-22.

[3] Sisto, D. J, Lachiewicz, P. F, \& Insall, J. N. Treatment of supracondylar fractures following prosthetic arthroplasty of the knee. Clin Orthop (1985). , 196, 265-72.

[4] Su, E. T, \& De Wal, H. Di Cesare P. Periprosthetic Femoral Fractures Above Total Knee Replacements. J Am Acad Orthop Surg (2004). , 12, 12-20.

[5] Hirsch, D. M, Bhalla, S, \& Roffman, M. Supracondylar fracture of the femur following total knee replacement. Report of four cases. J Bone Joint Surg Am (1981). , 63, 162-3.

[6] Lesh, M. L, Schneider, D. J, Deol, G, Davis, B, Jacobs, C. R, \& Pellegrini, V. D. The consequences of anterior femoral notching in total knee arthroplasty: a biomechanical study. J Bone Joint Surg Am (2000). , 82, 1096-101. 
[7] Dennis, D. A. Periprosthetic fractures following total knee arthroplasty: the good, bad and ugly. Orthopedics (1998). , 21, 1048-50.

[8] Scott, R. D. Anterior femoral notching and ipsilateral supracondylar femur fracture in total knee arthroplasty. J Arthroplasty (1988).

[9] Shawen, S. B. Belmont PJ Jr, Klemme WR, Topoleski LDT, Xenos JS, Orchowski JR. Osteoporosis and anterior femoral notching in periprosthetic supracondylar fractures. A biomechanical study. J Bone Joint Surg Am (2003). , 85, 115-21.

[10] Dennis, A. D. Periprosthetic fractures following total knee arthroplasty. J Bone Joint Surg Am (2001). , 83, 120-4.

[11] Ritter, M. A, Faris, P. M, \& Keating, E. M. Anterior femoral notching and ipsilateral supracondylar femur fracture in total knee arthroplasty. J Arthroplasty (1988). , 3, 185-7.

[12] Henry, S. L. Booth RE Jr. Management of supracondylar fractures above total knee prosthesis. Tech Orthop (1995). , 9, 243-52.

[13] Merkel, K. D. Johnson EW Jr. Supracondylar fracture of the femur after total knee arthroplasty. J Bone Joint Surg Am (1986). , 68, 29-43.

[14] Aaron, R. K, \& Scott, R. Supracondylar fracture of the femur after total knee arthroplasty. Clin Orthop (1987). , 219, 136-9.

[15] Dennis, D. A. Periprosthetis fractures following total knee arthroplasty. Tech Orthop (1999). , 14, 138-43.

[16] DiGioia AM 3d, Rubash HE. Periprosthetic fractures of the femur after total knee arthroplasty. A literature review and treatment algorithm. Clin Orthop (1991). , 271, $135-42$.

[17] Rorabeck, C. H, Angliss, R. D, \& Lewis, P. L. Fractures of the femur, tibia and patella after total knee arthroplasty: decision making and principles of management. Instr Course Lect (1998). , 47, 449-60.

[18] Rorabeck, C. H, \& Taylor, J. W. Classification of periprosthetic fractures complicating total knee arthroplasty. Orthop Clin North Am (1999). , 30, 209-14.

[19] Shatzker, J, \& Lambert, D. C. Supracondylar fractures of the femur. Clin Orthop (1979). , 138, 77-83.

[20] Insall, J. M. Fractures in the distal femur. In: Insall JM, editor. Surgery of the knee. New York: Churchill Livingstone, (1984). , 413-48.

[21] Hohl, M. Fractures about the knee. In: Rockwood CA, Green DP, editors. Fractures in adults.. Philadelphia: JB Lippincott,(1984). , 1478-9.

[22] Rorabeck, C. H, \& Taylor, J. W. Periprosthetic fractures of the femur complicating total knee arthroplasty. Orthop Clin North Am (1999). , 30, 265-77. 
[23] Harloww, M. L, \& Hofmann, A. A. Periprosthetic fractures. In: Scot WN, editor. The knee. St Louis: CV Mosby, (1994). , 1405-17.

[24] Sochart, D. H, \& Hardinge, K. Nonsurgical management of supracondylar fracture above total knee arthroplasty. Still the nineties option. J Arthroplasty (1997). , 12, 830-4.

[25] Ayers, D. C. Supracondylar fracture of the distal femur proximal to a total knee replacement. Instr Course Lect (1997). , 46, 197-203.

[26] Chmell, M. J, Moran, M. C, \& Scott, R. D. Periarticular fractures after total knee arthroplasty: principles of management. J Am Acad Orthop Surg (1996). , 4, 109-16.

[27] Figgie, M. P, \& Goldberg, V. M. Figgie HE III. The results of treatment of supracondylar fracture above total knee arthroplasty. J Arthroplasty (1990). , 5, 267-76.

[28] Kang Il KimEgol KA, Hozack WJ, Parvizi J. Periprosthetic fractures after total knee arthroplasties. Clin Orthop (2006). , 446, 167-75.

[29] Chen, F, Mont, M. A, \& Bachner, R. S. Management of ipsilateral supracondylar femuir fractures following total knee arthroplasty. J Arthroplasty (1994). , 9, 521-6.

[30] Sochart, D. H, \& Hardinge, K. Nonsurgical management of supracondylar fracture above total knee arthroplasty. Still the nineties option. J Arthroplasty (1997). , 12, 830-4.

[31] Engh, G. A, \& Ammeen, D. J. Periprosthetic fractures adjacent to total knee implants. Treatment and clinical results. J Bone Joint Surg Am (1997). , 79, 1100-13.

[32] Cusick, R. P, Lucas, G. L, Mcqueen, D. A, \& Graber, C. D. Construct stiffness of different fixation methods for supracondylar femoral fractures above total knee prosthesis. Am J Orthop (2000). , 29, 695-9.

[33] Lewis, P. L, \& Rorabeck, C. H. Periprosthetic fractures. In: Engh GA, Rorabeck CH, editors. Revision total knee arthroplasty. Baltimore MD: Williams \& Willkins; (1997). , 275-295.

[34] Kolb, K, Grützner, P. A, Marx, F, \& Kolb, W. Fixation of Periprosthetic Supracondylar Femur Fractures Above Total Knee Arthroplasty- The Indirect Reduction Technique with the Condylar Blade Plate and the Minimally Invasive Technique with the LISS. In: Fokter S, editor. Recent Advances in Hip and Knee Arthroplasty. Rijeka: InTech, (2012). , 315-42.

[35] Herrera, D. A, Kregor, P. J, Cole, P. A, Levy, B. A, Jonssos, A, \& Zlowodzki, M. Treatment of acute distal femur fractures above a total knee arthroplasty: systematic revew of 415 cases (1981-2006). Acta Orthop (2008). , 79, 22-7. 